\title{
ABHANDLUNGEN
}

\section{Staatsprojekt Europa}

\author{
Sonja Buckel
}

\section{State Project Europe}

Abstract: This essay offers a discourse analytical study of the trajectory of the European Court of Justice's case law regarding the social rights of non-employed persons, which began in 1998 and has established its centrepieces in the meantime. The text aims at demonstrating that societal struggles turn into legal ones, once they enter the arena of European law. I argue in particular that before the backdrop of the crisis of neoliberal hegemony the Court of Justice integrates different forces into a new hegemonic legal project, which points towards a European social union and provides first evidence of a potential 'state project Europe'. ${ }^{1}$

Keywords: European Integration, State and Legal Theory, Discourse Analysis, Transnational Social Rights

Schlagwörter: Europäische Integration, Staats- und Rechtstheorie, Diskursanalyse, transnationale soziale Rechte

\section{Einleitung}

Als María Martínez Sala, die zu diesem Zeitpunkt bereits seit 25 Jahren in der Bundesrepublik Deutschland lebte, 1993 beim Freistaat Bayern Erziehungsgeld beantragte, standen ihren Ansprüchen die beiden Exklusionsmechanismen des deutschen Wohlfahrtsstaats (nationale Zugehörigkeit und Erwerbszentrierung) entgegen: Zum einen war sie spanische Staatsbürgerin, zum anderen bezog sie Sozialhilfe. Demgemäß wurde ihr Antrag abgelehnt, was vom Sozialgericht Nürnberg bestätigt wurde. Doch zum gleichen Zeitpunkt - von der öffentlichen Diskussion relativ unbemerkt - waren UnionsbürgerInnenschaft und allgemeines Freizügigkeitsrecht im Maastricht-Vertrag verankert worden. Obwohl die meisten politischen und juristischen AkteurInnen darin nur symbolische Akte sahen, sollte dies alles ändern. Als nämlich das Bayerische Landessozialgericht dem Europäischen Gerichtshof die Frage vorlegte, ob die Regelung im Einklang mit dem

1 Für gemeinsame Diskussionen und Anregungen danke ich Andreas Fischer-Lescano, Lukas Oberndorfer, Margit Rodrian-Pfennig und Jens Wissel; für eine engagierte Kommentierung den beiden anonymen GutachterInnen; sowie für weiterführende Kritiken den Teilnehmenden des Workshops „Integration durch Recht“ während der Dreiländertagung der deutschsprachigen politikwissenschaftlichen Vereinigungen in Basel im Januar 2011, insbesondere Marcus Höreth. 
Recht der Europäischen Union stehe, begann dieser mit seinem Urteil im Jahr 1998 eine inzwischen über ein Jahrzehnt gefestigte Rechtsprechungslinie, ${ }^{2}$ die nichts Geringeres hervorbrachte als transnationale soziale Rechte nichterwerbstätiger UnionsbürgerInnen. Die Klägerin falle als Angehörige eines Mitgliedsstaats, die sich rechtmäßig im Gebiet der BRD aufhalte, in den Anwendungsbereich der Bestimmungen über die Unionsbürgerschaft und dürfe somit nicht aus Gründen der Staatsangehörigkeit diskriminiert werden, urteilte der Gerichtshof. ${ }^{3}$ In den folgenden Jahren kamen weitere Anspruchsberechtigte hinzu: Arbeitslose und Arbeitssuchende, Studierende und schließlich sogar ein Obdachloser. Der französische Staatsbürger Michel Trojani lebte zunächst 2001 auf einem belgischen Campingplatz und zog danach in ein Heim der Heilsarmee in Brüssel. Dort verdiente er sich mit Aushilfstätigkeiten im Rahmen eines Projekts der gesellschaftlichen Wiedereingliederung seine Unterkunft und ein geringes Taschengeld. Auch in diesem Fall lehnten die belgischen Sozialbehörden den Antrag auf ein Existenzminimum ab. Und wieder urteilte die große Kammer des Gerichtshofs, dass die Sozialhilfeleistung in den Anwendungsbereich des Vertrages falle, sich der Kläger rechtmäßig in Belgien aufhalte und daher nicht aufgrund der Staatsangehörigkeit diskriminiert werden dürfe. ${ }^{4}$

Unter GegnerInnen wie BefürworterInnen dieser Rechtsprechung ist unbestritten, dass sie einen „geradezu revolutionären Charakter" (Hilpold 2009, S. 41) aufweist. Indem der EuGH „ziemlich schnell Fleisch auf die Knochen der Unionsbürgerschaft brachte" (Meulman u. de Waele 2004, S. 275*5), konnte sich diese zum „notorischen Katalysator für die Vorschriften über die sozialen Rechte“ entwickeln (Lenaerts u. Heremans 2006, S. 103*), beziehungsweise zu einem „vom politischen Prozess autonomisierten Schwungrad“, mit dem „auf spektakuläre Weise der Zugriff des Gemeinschaftsrechts auf die nationalen sozialen Sicherungssysteme" vertieft werde (von Bogdandy u. Bitter 2005, S. 314).

Diese Entwicklung ist erstaunlich und wirft sozialwissenschaftlich grundlegende Fragen auf: Was treibt die RichterInnen des Europäischen Gerichtshofes an, eine solche Rechtsprechung zu entwickeln, die zudem den Vorstellungen der überwiegenden Mehrheit der Mitgliedsstaaten bei der Verabschiedung von UnionsbürgerInnenschaft und allgemeiner Freizügigkeit keineswegs entsprach (Wissel 2010)? Noch weiter gehend: Wie lässt sich diese Entwicklung im gesellschaftlichen Kontext der Europäischen Integration verstehen? Und schließlich: Wie erklärt sich die ,soziale' Rechtsprechung angesichts des Umstandes, dass der Gerichtshof seit Mitte der 1970er-Jahre viel eher das ,Schwungrad' des neoliberalen Binnenmarktprojektes war (Buckel u. Oberndorfer 2009; vgl. auch Höpner u. Schäfer 2008; Joerges 2010)?

Diesen Fragen möchte ich im Folgenden vor dem Hintergrund einer materialistischen Staats- und Hegemonietheorie und methodisch mit Hilfe einer hegemonie-

2 Beginnend mit der Rs. C-85/96, EuGH Slg. 1998 I-2691 - Martínez Sala, v. 12. Mai 1998 bis hin zur Rs. 22/08 und 23/08, EuGH Slg. 2009 I-0000, v. 04. Juni 2009.

3 Rs. C-85/98, Rn. 61-62.

4 Rs. C-456/02 Slg. 2004, I-7573, v. 07. September 2004, Rn. 41-44.

5 Alle nachfolgenden Literaturhinweise mit * sind Übersetzungen der Verfasserin. 
theoretisch inspirierten Diskursanalyse des Rechts nachgehen. ${ }^{6}$ Auf diese Weise lässt sich zeigen, wie gesellschaftliche Kämpfe auf dem rechtlichen Terrain ausgetragen werden. Das institutionelle Eigeninteresse der juristischen Instanz stellt sich dabei als Transformationsmechanismus dar, welcher konkurrierende gesellschaftliche Projekte in ein ,Staatsprojekt' übersetzt. Ich werde zunächst die theoretischen Prämissen meiner Untersuchungsperspektive erläutern (2.), danach die historisch-soziale sowie institutionelle Situiertheit dieses Rechtsdiskurses bestimmen (3.), um im Anschluss daran die Ergebnisse der Diskursanalyse vorzustellen (4.), die ich schließlich staats- und rechtstheoretisch interpretiere (5.).

\section{Staats- und rechtstheoretische Prämissen}

\subsection{Staatstheorie}

Das Spezifische materialistischer Ansätze ist der Versuch, moderne Staatlichkeit in Anlehnung an Nicos Poulantzas ([1978] 2002, S. 159) als „materielle Verdichtung gesellschaftlicher Kräfteverhältnisse" zu konzipieren. Dadurch sollen sowohl die gesellschaftlichen Kräftekonstellationen als auch die eigensinnigen Verfahren von Politik und Recht in den Blick genommen werden, in welchen die gesellschaftlichen Kräfteverhältnisse in politische und juridische übersetzt werden. Der moderne Staat kapitalistischer Gesellschaften wird auf diese Weise weder als Subjekt noch als Instrument mächtiger Akteure interpretiert, sondern vielmehr als eine strategisch-selektive Arena (Ludwig et al. 2009, S. 17). Weder besteht die ,Funktion' des Staates in der Lösung gesellschaftlicher ,Probleme', noch wird er als einheitliche Entität, als der Staat, aufgefasst. Vielmehr ist er ein soziales Verhältnis gesellschaftlicher Kräfte (Jessop 2007, S. 53), das diesen gegenüber allerdings eine verselbstständigte Form annimmt und dadurch über eine relationale Autonomie verfügt. Denn politische und ökonomische Herrschaft nehmen in der kapitalistischen Vergesellschaftung eine voneinander getrennte Form an, und diese Trennung bildet die Grundlage „des eigentümlichen institutionellen Aufbaus des Staates", sprich seiner Materialität (Poulantzas [1978] 2002, S. 47). Die gemeinsamen Angelegenheiten werden so von einer speziellen Instanz repräsentiert und von „dem Handlungszusammenhang der Staatsadministratoren“ (Lessenich 2009, S. 142) verwaltet. Das Allgemeine ist daher ein von der Gesellschaft abgesondertes Allgemeines. In politischen und rechtlichen Diskursen werden die widerstreitenden ,privaten` Interessen mit einem ,öffentlichen` Interesse vermittelt. „Der politische Kampf dreht sich im Kern um die Kontrolle über diese Universalisierungsinstanz“ (Jessop 2007, S. 51).

Konkrete Politiken (policies) werden als instabile Kompromisse zwischen gesellschaftlichen Kräften aufgefasst, die durch spezifische Staatsapparate formuliert werden. Da sich jeweils unterschiedliche Kräfte gemäß ihren Ressourcen in die Apparate einschreiben, ist die Heterogenität der Apparate ein zentrales Element

6 Die folgenden theoretischen und empirischen Darstellungen folgen den Ergebnissen des DFGgeförderten Forschungsprojekts „Die Transnationalisierung des Staates im Prozess des Entstehens einer gemeinsamen Europäischen Migrationskontrollpolitik“ (www.staatsprojekt-europa.eu). 
staatlicher Politiken (Brand 2011). Die Bürokratie ist ebenfalls keine einheitliche, sondern besteht aus verschiedenen Gruppen, Cliquen und Zentren, die vielfältige und verstreute Mikropolitiken verfolgen (Poulantzas [1978] 2002, S. 167). Die Strategien gesellschaftlicher Kräfte müssen also in die interne Struktur des politisch-administrativen Systems übersetzt werden. ${ }^{7}$

\subsection{Hegemonietheorie}

Für die Analyse der gesellschaftlichen Auseinandersetzungen um die Besetzung des Allgemeinen rekurriert materialistische Staatstheorie auf die Hegemonietheorie Antonio Gramscis. Er beschrieb mit Hegemonie einen wesentlichen Modus bürgerlicher Herrschaft, in welchem politische Führung voraussetzt, dass dominante gesellschaftliche Kräfte ihre eigenen ,korporativen' Interessen überschreiten, eine ,Katharsis' durchlaufen, durch die sie ein Bewusstsein von der Notwendigkeit der Berücksichtigung subalterner Interessen und damit der Universalisierung ihrer partikularen Position erlangen (Gramsci 1991 ff., H. 4, $\mathbb{S}$ 38, S. 495-496). Hegemonie ist die Fähigkeit, in diesem Prozess eine, Weltauffassung $^{6}$ zu entwickeln, einen ,common sense', der sich in allen gesellschaftlichen Bereichen manifestiert. Sie ist demnach eine Art zu leben und zu denken, sie bestimmt die Vorlieben, den Geschmack, die Mode sowie die moralischen und rechtlichen Normen. Sie organisiert das Verhältnis von Wissen und Wahrheit.

Dabei ist sie kein bloßer Reflex, sondern eine Organisationsleistung: Der kathartische Prozess der Entwicklung einer Weltanschauung muss organisiert werden. Diese Rolle hat Gramsci den sogenannten Intellektuellen zugeschrieben. Darunter sind zwar auch die ,großen Intellektuellen', die Literaten und Philosophinnen im klassischen Sinne zu verstehen, vor allem aber die ,kleinen Intellektuellen', also Partei- und Gewerkschaftsfunktionäre, Fernsehmoderatorinnen und Bürokraten, Journalistinnen und Think Tanks. Sie sind die TechnikerInnen der Hegemonie, die es vermögen, das Interesse eines komplizierten Allianzsystems auf den Begriff zu bringen und darüber eine Weltanschauung dezentral von verschiedenen gesellschaftlichen Orten aus herauszubilden. Dabei sind staatliche und rechtliche Verfahren aufgrund ihrer Formalisierung und der Herausbildung eigener ,Intellektueller' idealtypische Universalisierungsinfrastrukturen: Sie leisten in diesem Prozess die Übersetzung der Interessen gesellschaftlicher Kräfte in die internen Strukturen des Staates (vgl. Buckel u. Fischer-Lescano 2007).

Will man die gesellschaftlichen Auseinandersetzungen um Hegemonie empirisch bestimmen, muss man notwendig eine Reduktion von Komplexität vornehmen, da kausalanalytisch niemals jeder Beitrag eines jeden gesellschaftlichen Akteurs zu ermitteln sein wird. Daher bietet sich in Anlehnung an neogramscianische Autoren (van Apeldoorn 2009) das Konzept des Hegemonieprojektes an, in dem sich zumeist unbewusste Verknüpfungen einer Vielzahl unterschiedlicher Taktiken und Strategien verdichten, mittels derer versucht wird, die beteiligten partikularen

7 Für einen Überblick über aktuelle materialistische staatstheoretische Ansätze vgl. Demirović 2011; Esser 2008; Hirsch 2005; Lessenich 2009. 
Interessen in allgemeine zu transformieren, um hegemonial zu werden. Dass dieses Unterfangen auch scheitern kann, dass es umkämpft ist und sich immer im Prozess befindet, wird mit dem Begriff Projekt zum Ausdruck gebracht. Im Zentrum stehen, anders als bei der Interessengruppenforschung oder im Konzept der Advocacy-Coalition, die empirisch zu ermittelnden strategischen Praxen und nicht die Interessenspräferenzen bzw. die geteilten Wertvorstellungen. Allerdings müssen sich diese anonymen Strategien AkteurInnen zurechnen lassen, die wiederum sozialstrukturell positioniert sind und über entsprechende Ressourcen verfügen. Entlang der Strategien, der AkteurInnen, ihrer sozialstrukturellen Positionierung und ihrer Ressourcen lassen sich demnach verschiedene Hegemonieprojekte herausarbeiten, die immer schon auf einen strukturierten gesellschaftlichen Kontext bezogen sind.

Von den Hegemonieprojekten sind schließlich die hegemonialen Projekte zu unterscheiden: Gelingt es einem Hegemonieprojekt (als Verdichtung von strategischen Praxen), eine breite gesellschaftliche Zustimmung zu einer konkreten historischen Entwicklungsweise zu organisieren, so bildet sich kulturelle Hegemonie in einer spezifischen gesellschaftlichen Fragestellung heraus. Wird diese zudem in staatliche Politiken und juridische Normen übersetzt, handelt es sich um politische und juridische Projekte. Erst wenn dieser common sense sich in allen gesellschaftlichen Bereichen manifestiert und in den Alltagsroutinen reproduziert wird, ist ein spezifisches Projekt allgemein und damit hegemonial geworden.

\subsection{Staatsprojekt}

Unter den politischen Projekten spielt eines eine besondere Rolle: das ,Staatsprojekt'. Sein Effekt ist es, die Einheit des Staates zu gewährleisten, obwohl dieser ein heterogenes Ensemble von konkurrierenden Staatsapparaten ist, in welche sich die unterschiedlichen Kräfte einschreiben. Diese Einheit erschien im Nationalstaat des globalen Nordens selbstverständlich, die europäische Integration hingegen fördert ihre Unwahrscheinlichkeit zu Tage, da sie die vormals nationalen Staatsapparate reorganisiert. Das Staatsapparate-Ensemble des fordistischen Wohlfahrtsnationalstaats konnte durch ein Staatsprojekt, dessen zentrale Elemente die Nation und der Wohlfahrtsstaat waren, unter eine gewisse Einheit und Kohärenz gebracht werden (Jessop 1990, S. 128). In der Krise des Fordismus, Mitte der 1970er-Jahre, begann dieses jedoch aufzubrechen, während sich gleichzeitig ein neuartiges, mehrere soziale Räume durchkreuzendes, europäisches Ensemble von Staatsapparaten herausbildete, welches sowohl nationale (Ministerien, Grenzpolizeien etc.) als auch europäische Apparate (Kommission, EuGH, Agenturen wie Frontex etc.) umfasst. Der wesentliche Unterschied zum fordistischen Nationalstaat besteht nicht nur in der multiskalaren Ausdehnung, sondern vor allem darin, dass noch kein vergleichbares zusammenführendes europäisches Staatsprojekt existiert, während die tradierten nationalen Staatsprojekte weiterhin wirkmächtig sind. In der politikwissenschaftlichen Europaforschung hat sich für diese Konstellation die Bezeichnung der „Governance im Mehrebenensystem“ (vgl. etwa Tömmel 2007) etabliert. Allerdings, und das soll die vorliegende empirische Untersuchung verdeutlichen, ist ein mögliches Staatsprojekt im Entstehen. 


\subsection{Rechtstheorie}

In der vorgestellten Fallstudie soll der Beitrag des Europäischen Gerichtshofs zu einem solchen Staatsprojekt aufgezeigt werden. Dieser ist Teil des neuen Staatsapparate-Ensembles, folgt aber einer anderen Grammatik als die politischen Apparate. Politische Form und Rechtsform sind zwar aufeinander verwiesen, dennoch verfügen sie über jeweils eigene Verfahren, Intellektuelle und Regeln und - darüber vermittelt - über eine relationale Autonomie ${ }^{8}$, auch was ihr wechselseitiges Verhältnis betrifft. Sie sind raum-zeitlich verschieden institutionalisiert und bilden so eigensinnige Arenen gesellschaftlicher Auseinandersetzungen. Auch die Kämpfe um die sozialen Rechte wurden unter sehr verschiedenen strategisch-selektiven Bedingungen auf den beiden Terrains geführt. Die Rechtsform muss sich nicht a priori als rechtlicher Staatsapparat materialisieren; ist sie jedoch eingebunden in ein Ensemble unterschiedlicher politischer Apparate, das wie in der EU zumindest mittelbar über ein Gewaltmonopol verfügt, dann institutionalisiert sie sich als Ergebnis historischer Prozesse als rechtlicher Staatsapparat.

Das Konzept der Rechtsform begreift kurz gefasst (vgl. ausführlich Buckel 2007) das Recht kapitalistischer Gesellschaften als selbstreferenziellen Prozess aneinander anknüpfender Rechtspraxen: Gerichtsentscheidungen, Kommentar- und Fachliteratur, Rechtstheorien, Gutachten, Schriftsätze etc. Die gerichtlichen Verfahren bilden dabei das institutionelle Rückgrat. Ihre Verfahrensordnungen stellen eine „,objektive Grammatik` von Prozeduren, Kompetenzen, Entscheidungsund Argumentationsmodi“ bereit, die sie „auch zur Transformationsinstanz gesellschaftlicher Konflikte machen “ (Hitzel-Cassagnes 2006, S. 386). Die juristische Argumentation wirkt sich als Infrastruktur zur Universalisierung hegemonialer Projekte aus: Der Formzwang der abstrakten und tradierten Argumentationsfiguren und der dogmatischen Systematik zwingt zur Verallgemeinerung und Einbettung in den Kontext vergangener Entscheidungen. Vermittelt durch diesen Transformationsprozess kann es so einem oder mehreren Hegemonieprojekten gelingen, einen juridischen common sense, eine Vorstellung von dem, was rechtens ist, zu entwickeln. Das moderne Recht ist ein eigenständiger Ort gesellschaftlicher Hegemonieproduktion. Die juridischen Intellektuellen des Europäischen Gerichtshofs spielen im Gemeinschaftsrecht eine zentrale Rolle, denn sie werden zu OrganisatorInnen eines feingliedrigen rechtsförmigen Konsenses: Sie fügen die aufeinandertreffenden Interessen in Form von Durchbrüchen und darauf folgenden Zugeständnissen in einem langwierigen Prozess zusammen. Wie ich anschließend zeigen werde, muss es ihnen gelingen, eine dogmatische Argumentation zu entwickeln, die sich sowohl kohärent in das bisherige System einfügt, als auch die unterschiedlichen gesellschaftlichen Interessen miteinander vermittelt. Das Recht kapitalistischer Gesellschaften wird in der materialistischen Tradition als ,soziale Form' verstanden, das heißt als verdinglichter Handlungszusammenhang, der sich gegenüber den gesellschaftlichen AkteurInnen verselbstständigt (vgl. Buckel 2007:

8 Ich verwende hier anstelle des aus der marxistischen Rechtstheorie stammenden Begriffs der „relativen Autonomie“ den der „relationalen Autonomie“. Dies soll auf bestimmte Missverständnisse dieser Verwendung hinweisen und eine Alternative bieten; ausführlich Buckel 2007: 242-245. 
230-250). Dadurch verfügt die Rechtsform über eine ,relationale Autonomie', und die rechtliche Bürokratie kann eine institutionelle Eigenlogik entwickeln ähnlich dem von Offe so bezeichneten „Interesse des Staates an sich selbst“ - (vgl. dazu Lessenich 2009, S. 144; Esser 2008, S. 215). Dieses Bestandsinteresse wird jedoch wiederum über die Fähigkeit, einen vorübergehenden asymmetrischen Konsens herauszubilden vermittelt, weil andernfalls die Legitimation der Institution selbst infrage gestellt werden könnte.

\section{Situiertheit des juridischen Diskurses der transnationalen sozialen Rechte}

Ich werde der theoretischen Darstellung folgend nun zunächst den Kontext des Diskurses skizzieren. Dabei soll dem Konzept der materiellen Verdichtung gesellschaftlicher Kräfteverhältnisse Rechnung getragen werden. Somit beginne ich mit der gesellschaftlichen Kräftekonstellation - operationalisiert in Hegemonieprojekte - und schließe daran mit der institutionellen Ausgangssituation auf dem politischen und rechtlichen Terrain an, um daraufhin mithilfe der Diskursanalyse zu zeigen, wie diese gesellschaftliche Auseinandersetzung in der Rechtsform ausgetragen wurde. Die Darstellung der Hegemonieprojekte folgt dabei zum einen Erkenntnissen der neogramscianischen Politischen Ökonomie und zum anderen basiert sie auf exemplarischen Sozialstrukturanalysen der BRD, Großbritanniens und Spaniens. Diese können an dieser Stelle nur zusammenfassend dargestellt werden (für eine ausführliche Erläuterung vgl. Kannankulam et al. 2011) und sind aufgrund der mit dem Ansatz verbundenen Komplexität als heuristische Idealtypen zu betrachten.

\subsection{Die gesellschaftliche Kräftekonstellation: Hegemonieprojekte in der Krise der wettbewerbsstaatlichen Integrationsweise}

Waren das Binnenmarktprojekt und die Währungsunion zentrale neoliberale hegemoniale Projekte, welche die Europäische Integration vorantrieben (Bieling u. Steinhilber 2000), so geriet diese bereits Mitte der Neunzigerjahre in die Krise: Wirtschaftliche Stagnation und steigende Arbeitslosenzahlen ließen weitere monetäre Integrationsschritte nicht mehr attraktiv erscheinen. Erste Anzeichen einer Legitimationskrise der EU zeigten sich darin, dass größere Teile der Bevölkerung immer weniger bereit waren, die sozialen Konsequenzen der neoliberalen Restrukturierung zu akzeptieren (Bieling u. Steinhilber 2000, S. 118). Diverse Proteste kristallisierten sich zunächst in sozialdemokratischen Wahlerfolgen und Forderungen nach einem sozial verantwortlichen politischen Europa. Ende der 1990er-Jahre, also zu Beginn der zu untersuchenden EuGH-Rechtsprechung, begann sich die Krise dann zu einer „Mehrebenen-Legitimationskrise“ (van Apeldoorn 2009, S. 21*) zu verstärken, welche die Grundlagen der wettbewerbsstaatlichen Integrationsweise (Ziltener 1999, S. 200) infrage stellte. Diese Situation spitzt sich seit 2008 durch die Weltwirtschaftskrise zu. Angesichts des Fehlens eines kohärenten alternativen Projekts führt die Krise aber nicht zum Ende der europäischen neoliberalen Politiken. Sondern - wie die aktuelle Währungs- und Staatsschuldenkrise zeigt - sowohl die ausbleibende Harmonisierung der Wirt- 
schaftspolitik als auch die fehlende Europäisierung der Sozialpolitik (jenseits regulativer Politiken) wirken sich krisenverstärkend aus. Das erhebliche Wohlstandsgefälle innerhalb Europas und damit verbunden die divergenten Wohlfahrtssysteme erzeugten die Widerstände gegen eine EU-Sozialpolitik. Hinzu kommt das Fehlen eines eigenen nennenswerten Budgets der EU, mit dem dieses Gefälle ausgeglichen werden könnte (Leibfried u. Obinger 2008, S. 338-339). Die „Konstruktion einer sozialstaatlich nicht eingehegten Marktintegration“ verhärtet sich so und „kann zu einer ernsten Gefahr für das Integrationsprojekt selbst werden “ (Leibfried u. Obinger 2008, S. 340-341).

In dieser Konstellation ringen fünf europäische Hegemonieprojekte um den Fortgang der Europäisierung: (a) Das inzwischen nicht mehr hegemoniale sondern nur noch dominante Projekt ist das neoliberale. Es verdichten sich hierin diejenigen Strategien, die auf die Transnationalisierung von (Finanz-)Kapital, Handel, Produktions- und Reproduktionsketten setzen sowie auf die Mobilität des Produktionsfaktors Arbeit im Kontext einer neokolonialen internationalen Arbeitsteilung. Die Akteure dieses Projekts sind die Schlüsselsektoren des globalisierten, postfordistischen Akkumulationsregimes: die Finanzwirtschaft und die großen transnationalen Konzerne, jeweils repräsentiert durch ihre Verbände. Die Ressourcen des neoliberalen Hegemonieprojekts zur Durchsetzung der Ziele sind außerordentlich hoch und somit auch die Konfliktfähigkeit der Akteure. (b) Für die Strategien des national-sozialen Hegemonieprojekts stehen demgegenüber sozialer Ausgleich und soziale Umverteilung im Mittelpunkt. Zentral ist die (notgedrungene) Verteidigung etablierter nationaler Sozialsysteme, um angesichts des bestehenden neoliberalen Globalisierungsdrucks sozialpolitische Errungenschaften zu schützen. Dem liegt die politische Einschätzung zugrunde, dass eine Strategie der Europäisierung geringe Erfolgsaussichten hat. Die soziale Basis dieses Projekts besteht in den Resten der Kräfte der fordistischen Produktionsweise, aus denen sich Finanzindustrie und transnationale Konzerne im Zuge der Globalisierungsprozesse herausgelöst haben. Zwar sind die Ressourcen dieser Kräfte geschrumpft, doch die AkteurInnen des national-sozialen Projekts sind noch immer konfliktfähig, vor allen Dingen die relevanten Teile der gewerkschaftlich organisierten ArbeitnehmerInnenschaft. Davon ist (c) ein national-konservatives Hegemonieprojekt zu unterscheiden, welches einem politisch vertieften europäischen Integrationsprozess skeptisch bis ablehnend gegenübersteht und ein ,Europa der souveränen Nationen' befürwortet. Es assoziiert die Nation mit Ethnizität, gemeinsamer Sprache, Geschichte, Kultur und bestimmten konservativen Werten wie Familie, einem traditionellen Geschlechterrollenbild, Heimat, Sicherheit und christlicher Religion. Die soziale Basis des national-konservativen Projekts bilden vor allem Sektoren, die am stärksten von Konkurrenz durch internationale Konzerne oder grenzüberschreitende Dienstleister bedroht sind, da sie hauptsächlich oder ausschließlich national beziehungsweise lokal ausgerichtet sind. Dazu gehören außerdem Teile der ,weißen' Arbeiterklasse und der unteren Mittelschicht. Diese Akteure gehen in vielen Ländern im Rahmen konservativer Parteien und Stiftungen enge Bündnisse mit Akteuren des neoliberalen Hegemonieprojekts ein und profitieren von deren Ressourcen. (d) Des Weiteren lassen sich AkteurInnen identifizieren, deren Strategien auf die Rettung des europäischen Sozialmodells 
durch eine gesamteuropäische Sozialpolitik zielen. Ihre Taktiken und Strategien verdichten sich im proeuropäisch-sozialdemokratischen Hegemonieprojekt. Die Grundannahme ist, dass der Krise des Wohlfahrtsstaates im Kontext neoliberaler Globalisierungsprozesse nur durch eine Europäisierung der Sozialpolitik sowie eine europäische Tarifpolitik begegnet werden kann. Ein ,soziales Europa' wird als Gegenkonzept in Stellung gebracht. Die soziostrukturelle Positionierung dieses Projekts ist vergleichsweise schwach. Einerseits vertreten viele europäische Gewerkschaften eine äußerst proeuropäische Haltung. Eine wichtige Rolle spielen auch jene Teile der sozialen Bewegungen, die unter dem Slogan „Another Europe is possible" eine anti-neoliberale und zugleich proeuropäische Position vertreten. Dabei besteht allerdings ein großes strategisches Problem: Trotz ihrer prinzipiell proeuropäischen Ausrichtung sind sie durch die existierenden politischen Strukturen gezwungen, weitgehend auf nationaler Ebene zu agieren. Das proeuropäische sozialdemokratische Hegemonieprojekt ist jedoch ein wichtiger Bündnispartner der Kommission, weil diese darüber ihre eigene institutionelle Position als genuin europäische Akteurin stützen und ausbauen kann. Abschließend lässt sich (e) ein linksliberal-alternatives Hegemonieprojekt identifizieren. Es ist Träger eines politischen Liberalismus, der Toleranz mit Menschenrechten verbindet. Das Projekt basiert auf den AkteurInnen des politischen Liberalismus und der Neuen Linken, solchen, die aus der dritten Welle der Frauenbewegung kommen, sich für Antirassismus und Umweltschutz einsetzen. Die soziale Basis sind sowohl gebildete Prekarisierte als auch etablierte bildungsbürgerliche Schichten. Entsprechend den postnationalen Werten und teilweise postmateriellen Interessen dieser Milieus vertreten seine AkteurInnen eine ausgesprochen proeuropäische und internationalistische Strategie, welche die EU als Konstellation postnationaler BürgerInnenschaft ansieht. Ihre ökonomischen Ressourcen sind vergleichsweise schwach, gleichwohl stellen die Ausweitung des linksalternativen Milieus bis in bürgerliche Schichten hinein und die Unterstützung durch verschiedene Öko-, Technologie- und Kreativbranchen eine nicht zu ignorierende Basis dar. Die besonderen Ressourcen dieses Projekts liegen im hohen sozialen und kulturellen Kapital.

Im dritten Abschnitt werde ich nachzeichnen, wie diese gesellschaftlichen Kräfte diskursiv den juridischen Konflikt um das Allgemeine der europäischen Sozialpolitik geführt haben. Zuvor müssen aber noch die Ausgangsbedingungen des politischen und des juridischen Terrains skizziert werden, auf denen die Auseinandersetzungen stattfanden.

\subsection{Das politische Terrain: Vom Marktbürger zur Unionsbürgerin}

Der Positivierung von UnionsbürgerInnenschaft und Allgemeinem Freizügigkeitsrecht mit dem Vertrag von Maastricht 1992 waren langwierige politisch-institutionelle Auseinandersetzungen im Rat unter den Mitgliedsstaaten aber auch mit der Europäischen Kommission und dem Europäischem Parlament vorausgegangen (ausführlich Wissel 2010). Während Spanien, Frankreich und die BRD vehemente Befürworterinnen waren, opponierte vor allem Großbritannien unter Thatcher, die einen Verlust nationaler Souveränität befürchtete (Wissel 2010, S. 36-40), wohingegen wiederum sowohl Kommission als auch Parlament und Teile der Zivil- 
gesellschaft diese Regelungen viel früher schon gefordert und weitergehende Vorschläge unterbreitet hatten.

Nachdem die Kommission 1985 in ihrem Binnenmarkt-Weißbuch (KOM[85] 310) gefordert hatte, dass Freizügigkeit nicht ausschließlich von der Arbeitskraft abhängen dürfe, wurden schließlich Anfang der 1990er-Jahre im Ministerrat drei Richtlinien zur Freizügigkeit von Studierenden, RentnerInnen, Arbeitssuchenden und anderen Nichterwerbstätigen beschlossen. ${ }^{9}$ Die Besonderheit lag darin, dass die Rechtsansprüche dieser Personengruppe nicht auf die Grundfreiheiten zurückgeführt werden konnten. Daher war auch bis zum Schluss umstritten, ob es überhaupt eine vertragliche Grundlage für die Richtlinien gab. Die Verhandlungen gestalteten sich insbesondere in Bezug auf die sozialen Leistungen kompliziert (Wind 2009, S. 226). Letztlich einigte man sich darauf, dass Nichterwerbstätigen zwar Freizügigkeit ermöglicht werden sollte, nicht aber der Zugang zu öffentlichen Leistungen des Aufnahmestaates. Der Maastricht-Vertrag brachte dann den „qualitativen Sprung“ (Renner 2005, Vorbem. Rdnr. 3), nämlich die zentrale institutionelle Veränderung auf der Ebene der europäischen Verträge durch die Einführung der UnionsbürgerInnenschaft in Art. 17 EG (Art. 20 AEUV) und des Freizügigkeitsrechts in Art. 18 EG (21 AEUV). ${ }^{10}$ Dabei wurde Art. 18 EG (Primärrecht) unter den Vorbehalt der nachrangigen Richtlinien (Sekundärrecht) gestellt: Nur unter den „Bedingungen und Beschränkungen“ (Abs. 2) etwa der drei Nichterwerbstätigen-Richtlinien (und damit des Erfordernisses ausreichender Existenzmittel) sollte das Freizügigkeitsrecht gelten. Die Möglichkeit zur Beschränkung des Zugangs zu den nationalen sozialen Leistungssystemen sollte demnach beibehalten werden.

Auf dieser vertraglichen Grundlage wurde dann 2004 die neue FreizügigkeitsRichtlinie erlassen, die den bisherigen „wahren Flickenteppich“ (Schönberger 2006, S. 226) der diversen Freizügigkeits-Richtlinien für Erwerbstätige und Nichterwerbstätige systematisch zusammenfasste und ersetzte. ${ }^{11}$ „Eine grundlegende Neuorientierung enthielt die Richtlinie dabei insofern, als sie das Aufenthaltsrecht der Unionsbürger - ungeachtet der Art ihrer Betätigung - nach einem fünfjährigen rechtmäßigen Aufenthalt in einem anderen Mitgliedsstaat in ein sehr verfestigtes Daueraufenthaltsrecht einmünden ließ“ (Schönberger, S. 227). Dieses unterschied sich kaum mehr von dem der jeweiligen StaatsbürgerInnen. Damit war es zu einem grundlegenden Statuswandel der europäischen BürgerInnen gekommen. Nachdem sie vierzig Jahre lang nur MarktbürgerInnen oder ,GastarbeiterInnen' gewesen waren, deren Rechtsstellung sich einzig aus den Grundfreiheiten (etwa der ArbeitnehmerInnenfreizügigkeit) ableitete, erhielten sie nun einen

9 RL 90/365/EWG des Rates vom 28.06.1990 über das Aufenthaltsrecht der aus dem Erwerbsleben ausgeschiedenen Arbeitnehmer und selbstständig Erwerbstätigen, ABlEG 1990 Nr. 1 180, S. 28; RL 93/96/EWG des Rates vom 29.10.1993 über das Aufenthaltsrecht der Studenten, ABlEG 1993 Nr. L 317, S. 59; RL 90/364/EWG des Rates über das Aufenthaltsrecht vom 13.07.1990, ABIEG 1990 Nr. L a 180, S. 26.

10 Was die zentralen Normen des zu analysierenden Rechtsdiskurses betrifft, wird im Folgenden nicht die Nummerierung des Lissabon-Vertrags (das heißt des Vertrages über die Arbeitsweise der EU) verwendet, sondern die Nummerierung, die zum Zeitpunkt der Rechtsprechung die maßgebliche war: die des EG-Vertrags nach Amsterdam.

11 RL 2004/38/EG, ABlEU. L 158/77, v. 30.4.2004. 
politischen Bürgerschaftsstatus. Die EU war Anfang der 1990er-Jahre auf dem Sprung von einer bloß ökonomischen Wirtschaftsgemeinschaft zur politischen Union. Die „Platzierung von Bürgerschaft jenseits des Staates“, die „die konstante Größe einer durch das Territorialprinzip definierten politischen Gemeinschaft unterminiert" (Wiener 2005, S. 222), war zugleich der erste Durchbruch des Staatsprojekts Europa auf dem politischen Terrain.

\subsection{Das juridische Terrain: "The awakening of the ECJ's social awareness"12}

Nach dem Inkrafttreten des Maastrichter Vertrags begann zunächst ein noch nicht rechtlich institutionalisierter Vordiskurs über Inhalt und Bedeutung der neuen Normen. Dabei war die anfängliche Haltung der juristischen Literatur ganz überwiegend „von kritischer Distanz, ja zum Teil von herablassender Missachtung geprägt. Die emphatische Natur des Begriffs präsentierte sich eigenartig vor dem Hintergrund eines bescheiden erscheinenden Regelungsgehalts" (Hilpold 2008, S. 17). Joseph Weiler etwa sah in der UnionsbürgerInnenschaft „wenig mehr denn eine zynische Werbeaktion" (Weiler 1996*), denn sie beinhaltete scheinbar keine neuen Rechtspositionen, erschien eher als symbolische Politik, als halbherziger Legitimationsversuch angesichts des Demokratiedefizits. In dieser Enttäuschung artikulierte sich eine spezifische Kräftekonstellation: Hinter der Einführung der UnionsbürgerInnenschaft, die unter dem Banner des „Europas der Bürger" schon seit Beginn der Gemeinschaft immer wieder gefordert und seit den 1970er-Jahren auch politisch-institutionell durch Kommission und Europäisches Parlament voranzutreiben versucht wurde (Wissel 2010, S. 16-22), standen keine starken Interessen, sondern sie ging vielmehr einerseits auf Forderungen von wenigen NGOs, dem Europäischen Gewerkschaftsbund, vereinzelten Bürgerrechtsgruppen und europafreundlichen Intellektuellen sowie andererseits auf Hoffnungen der südlichen Staaten zurück, durch eine politische Union der Heterogenität der EG und der damit drohenden eigenen Marginalisierung als europäische Peripherie entgegenwirken zu können (Wissel 2010, S. 3-4). So erfolgte die vertragliche Normierung maßgeblich auf Drängen des damaligen spanischen Ministerpräsidenten Felipe González (Wissel 2010, S. 33-35 + 39-43). Die über Jahrzehnte ausgetragenen Kämpfe um die UnionsbürgerInnenschaft waren mit ihrer Positivierung im EG-Vertrag auf dem politischen Terrain zu einem vorläufigen Ende gekommen. Die BefürworterInnen hatten sich durchgesetzt, aber um den Preis, dass ihre Ausformulierung für die meisten politischen Beteiligten nicht viel mehr als die Bestätigung des ohnehin schon vorhandenen Acquis darstellte (Wissel 2010, S. 38). Die gegensätzlichen Positionen, die eine weitergehende BürgerInnenschaft forderten beziehungsweise diese ganz ablehnten, existierten damit fort. Dies führte zu einer zunächst eher symbolischen Positivierung. Die Auseinandersetzungen verschoben sich nun auf ein anderes, das juristische Terrain.

Dort angekommen, vermieden es die RichterInnen des Gerichtshofes jedoch in den ersten Jahren, auf die neue UnionsbürgerInnenschaft auch nur Bezug zu neh-

12 Hatzopoulos 2005, S. 1604. 
men. Auch der Allgemeinen Freizügigkeit in Artikel 18 EG Abs. 1 wurde nur eine marginale Rolle zuerkannt (Hilpold 2008, S. 20). „Wagemutigere Sondierungsversuche" (Hilpold 2008, S. 20) unternahmen hingegen schon früher die GeneralanwältInnen. Sie begründeten eine Tradition, „den grundrechtsgeschützten Bürgerschaftsdiskurs mit Verve weiterzuentwickeln" (Haltern 2005, S. 92). Doch die RichterInnen waren „noch nicht bereit, auf dem noch weitgehend unergründeten Feld der Unionsbürgerschaft" den weitreichenden Thesen zu folgen (Hilpold 2008, S. 21). Sie blieben noch eine Weile auf ihrem bisher etablierten Pfad der extensiven Ausweitung des ArbeitnehmerInnenbegriffs und subsumierten mögliche Ansprüche unter die ArbeitnehmerInnen-Freizügigkeit (Wind 2009, S. 250). Erst 1998 begann die Hinwendung zur UnionsbürgerInnenschaft: „Sein Weg führte [den EuGH] von völliger Ignorierung über eine tastende Suche nach Potential zu einer Konzeptionalisierung, die die Unionsbürgerschaft von einer rechtlichen Marginalie zu einer festen und dynamischen Größe hat werden lassen“ (Haltern 2005, S. 95).

\section{Diskursanalyse}

\subsection{Hegemonietheoretische Diskursanalyse des Rechts}

Um nun die hegemonialen Auseinandersetzungen im Recht zu untersuchen, bietet sich ein diskursanalytisches Vorgehen an, welches um hegemonietheoretische Erkenntnisse erweitert wurde, so wie dies Martin Nonhoff vor allem im Anschluss an Michel Foucault und Ernesto Laclau für die Diskursanalyse des Politischen vorgeschlagen hat. Aufgrund der eigenen Grammatik des Rechtlichen müssen diese Überlegungen jedoch rechtstheoretisch angepasst werden. Die Textgattung ist eine andere als diejenige politisch-programmatischer Schriften, Rechtstexte werden in anderen Verfahren von spezifisch juristisch geschulten AkteurInnen formuliert und folgen anderen Formationsregeln. Im Zentrum des Rechtlichen steht die juristische Argumentation: die etablierten Rechtsfiguren als Ablagerungen vergangener Auseinandersetzungen, welche aufgegriffen und verschoben werden. Die konflikthaften Auseinandersetzungen um das Allgemeine (Nonhoff 2006, S. 109) sind auch für das Recht entscheidend, aber eben nicht als programmatische Forderungen nach einem erst zu schaffenden Allgemeinen, sondern als Kampf um die etablierte normative Ordnung, die das Allgemeine bereits zu enthalten beansprucht und es nur noch, scheinbar rein technisch, in Einzelfallentscheidungen zu verwirklichen vorgibt. Die Auseinandersetzungen um Hegemonie finden in der Rechtsform also subtiler, immer vermittelt über die Auslegung von rechtlichen Figuren, statt.

Noch an einer zweiten Stelle weiche ich von Nonhoffs Konzeption ab, nämlich an der Bestimmung des Allgemeinen selbst. Nonhoff folgt Laclau und fasst darunter das Vollkommene, Allen-gerecht-Werdende, das notwendig einen „leeren Ort“ bilden müsse. Dies sei eine universelle Eigenschaft einer sozialen und politischen Ontologie: der „konstitutive Mangel von Gesellschaft“ (Nonhoff 2006, S. 116). Das Allgemeine ist danach niemals greifbar und muss durch eine Repräsentation seiner selbst supplementiert werden. Es wird zu einem Äquivalent, „zu einem ho- 
rizonthaften Imaginären einer vom Allgemeinen durchdrungenen idealen Gesellschaft" (Nonhoff 2006, S. 117) Auch wenn ich die Beschreibung teile, so doch nicht die psychoanalytische Begründung: Die Bereiche des Imaginären und Symbolischen sind Konzepte Lacans der individuellen Psyche, die Laclau und mit ihm Nonhoff auf politische Gemeinschaften übertragen und sie so „in die politische Theorie inkorporieren“ (Nonhoff 2006, S. 111). Dies ist methodisch fragwürdig. Der Soziologe und Psychoanalytiker Alfred Lorenzer hatte bereits gegen den Freudomarxismus eingewandt, dass die Psychoanalyse sich über die Reichweite ihrer Kategorien bewusst werden und sich methodisch beschränken müsse: Die Resultate einer Analyse der Subjektivität und diejenigen der gesellschaftlichen Strukturen müssen aufeinander bezogen werden, ohne die beiden Untersuchungsperspektiven ineinander aufzulösen (ausführlich Buckel u. König 2009). Die Übertragung psychischer Strukturen auf politische Gemeinschaften führt andernfalls zu einer ahistorischen Ontologie. Universalisierung ist gerade eine historisch-spezifische, erst mit der bürgerlichen Gesellschaft und ihrem Staat entstandene Praxis. Der ,Mangel' am Allgemeinen entsteht dadurch, dass, obwohl dieses zum zentralen Referenzpunkt wird, „wahre Allgemeinheit“ aufgrund der vielfältigen Herrschaftsverhältnisse nicht möglich ist (Neumann 1937, S. 594) und der Staat eine gesonderte, vom Ökonomischen getrennte Form annimmt (s. o.). So werden permanent hegemoniale Auseinandersetzungen um das Allgemeine geführt, und nur vorübergehend und niemals unbestritten kann eine hegemoniale Konstellation erfolgreich behaupten, die Vorstellung der gerechten Gesellschaft zu repräsentieren.

Die strategischen Praxen lassen sich im Diskurs als ,Strategeme' untersuchen, wobei die Rechtsfiguren die maßgeblichen Analysekategorien bilden. Nonhoff (2006) hat für die Identifizierung eines hegemonialen Projektes die Anwesenheit von drei „Kernstrategemen der Hegemonie“ (Nonhoff 2006, S. 214) benannt.

Zum Ersten die Äquivalenzierung: Wenn Hegemonie bedeutet, dass eine Position über diejenigen Interessen hinaus, von denen sie artikuliert wird, anschlussfähig ist, muss sie eine Vielzahl von Subjektivierungsangeboten machen (Nonhoff 2006, S. 214). Diskurstheoretisch lässt sich das in der Verknüpfung von differenten Positionen als Äquivalente, die gemeinsam für das Gesamtprojekt - das Allgemeine - stehen, analysieren: als, Äquivalenzkette‘. Im Unterschied dazu verlangt das zweite Strategem eine antagonistische Zweiteilung des Raums, die Benennung all derjenigen diskursiven Elemente, zu denen die Äquivalenzbeziehung in Kontrarität steht. Die „pluralistischen Konflikte um die Besetzung des leeren Orts des Allgemeinen“ spitzen sich „auf eine klare Alternative“ zu (Nonhoff 2006, S. 222). Beim dritten Kernstrategem handelt es sich schließlich um die Repräsentation des Allgemeinen. Eine bestimmte äquivalente Position wird zum „exklusiven Makler", vermittelt zwischen den anderen Positionen und versucht so, das Allgemeine zu repräsentieren (Nonhoff 2006, S. 218). Daneben gibt es weitere ergänzende Strategeme, die nicht notwendig sind, um eine diskursive Praxis als hegemoniale zu kennzeichnen, die aber dennoch zentral sind, weil sie die Reichweite einer hegemonialen Formation erhöhen (Nonhoff 2006, S. 233-234). Zwei solcher spezifisch juridischer Strategeme, das Ausweich- und das Zugeständnisstrategem, werde ich im Folgenden darstellen. Schließlich sind noch zwei Arten von hegemonialen Strategien voneinander zu unterscheiden: die offensiv- und die de- 
fensiv-hegemoniale Strategie. Erstere sucht Hegemonie zu etablieren, während für die zweite das Nichtvorhandensein eines Projektes kennzeichnend ist: Sie setzt daher auf Abwehr und Zerstreuung des hegemonialen Projekts (Nonhoff 2006, S. 238).

Der Materialkorpus der untersuchten Fallstudie besteht aus den bisher wesentlichen zwölf Urteilen des Europäischen Gerichtshofes zu den sozialen Rechten Nichterwerbstätiger, den Schlussanträgen der GeneralanwältInnen, Kommentarliteratur, den Aufsätzen darüber in europäischen Fachzeitschriften, Lehrbüchern und Monografien sowie aus der Berichterstattung europäischer Tageszeitungen. Diesen Prozess werde ich nun im Folgenden entlang der Strategeme nachvollziehen und beginne zunächst - zur Identifizierung des hegemonialen Projektes - mit den drei Kernstrategemen.

\subsection{Unionsbürgerschaft als Repräsentantin des Allgemeinen}

In dem zu untersuchenden Diskurs artikulieren sich zwei antagonistische Konzeptionen des Allgemeinen: eine proeuropäische und eine nationale. Dabei verfolgt die proeuropäische Diskurskoalition eine offensiv-hegemoniale, die nationale hingegen eine defensiv-hegemoniale Strategie. Erstere besteht aus einem Amalgam von Strategien des proeuropäisch-sozialdemokratischen und des linksliberal-alternativen Hegemonieprojekts. Die Normen werden interpretiert als „universelles Anti-Diskrimierungsrecht inklusive des Zugangs zu allen Arten von Wohlfahrtsleistungen im Gemeinschaftsrecht“. Dabei werde die EU zur „,angemessen ,Gemeinschaft ${ }^{\star}$, in der soziale Probleme, inklusive der Verteilung von öffentlichen Gütern [...] gelöst werden müssen“, zu einer „Körperschaft aller EU-BürgerInnen" anstelle bloß separater nationaler Bürgerschaften (Shaw 1998, S. 536, 556*). Aus Art. 18 EG folgten unmittelbar nach diesem „maximalistischen Ansatz" nicht nur das Aufenthaltsrecht sondern „auch alle damit im Zusammenhang stehenden oder daraus ableitbaren Rechte" (Borchardt 2000, S. 2059). Die Angehörigen der EU seien durch die Unionsbürgerschaft, so die portugiesische Regierung ${ }^{13}$ in ihrer Erklärung zur Rechtssache Grzelczyk ${ }^{14}$, nicht länger „bloße Wirtschaftsfaktoren“. Vielmehr „haben die Mitgliedsstaaten ihren politischen Willen bekundet, „ein Europa der Bürger voranzutreiben“ (Letzner 2003, S. 119, Herv. i. O.). Das Ende einer Ära sei eingeleitet, „in der Mitgliedsstaaten den BürgerInnen anderer Mitgliedsstaaten den Zugang zu ihren wohlfahrtstaatlichen Leistungen einzig auf Grund ihrer Nationalität verweigern konnten. [...] Im Prinzip verlangt das Gemeinschaftsrecht die De-Nationalisierung der Europäischen Wohlfahrtstaaten“ (van der Mei 2005, S. 207*). Die Rechtsprechung des EuGH ermögliche also „transnationale Solidarität“ (Barnard 2005, S. 1477*) und habe

13 Nach Art. 40 der Satzung des EuGH können die EU-Organe und Mitgliedsstaaten einem anhängigen Rechtsstreit beitreten.

14 Rs. C-184/99 vom 20.09.2001, Slg. 2001, I-6193. Wiedergegeben nach den Schlussanträgen v. GA Alber, v. 28.09.2000, Rdnr. 52. Der französische Staatsbürger Rudy Grzelczyk studierte in Belgien und beantragte zum Ende seines Studiums das belgische Existenzminium, welches aber nur belgischen StaatsbürgerInnen oder EU-ArbeitnehmerInnen zustand. 
daher einen „viel politischeren, humaneren, sozialeren und solidarischeren Charakter" als die einstigen Römischen Verträge (Candela Soriano 2005, S. 1477*). Die Konzeption des Allgemeinen der defensiv-hegemonialen Strategien besteht hingegen in keiner eigenen Vision, sondern in einer Verneinung der proeuropäischen Konzeption. Die EG habe „noch nicht die Schwelle zu einer wahren Sozialunion überschritten, in der die Völker [...] als eine einzige Gemeinschaft betrachtet werden, die gegenseitig Solidarität ausweiten, in der Einkommen und finanzielle Belastungen geteilt werden, unabhängig von nationalen Grenzen ". Sozialleistungen würden durch die Teilnahme am „kollektiven Arbeitsprozess“ verdient, so die national-soziale Position, und seien daher die „Crux der Freizügigkeit" (Tomuschat 2000, S. 454*). Der "ausgeprägte Solidaritätsgedanke“, der dem Unionsbürgerschaftskonzept zwar durchaus innewohne, sei aber im Gemeinschaftsrecht nicht verankert worden. Die Unionsbürgerschaft sei lediglich eine „Klammer über bereits Gegebenes“ gewesen, „ohne unmittelbar einen Mehrwert hinzuzufügen “. Es könne daher nicht angehen, dass der EuGH die nicht-wirtschaftliche Bewegungsfreiheit weitreichend verselbstständige, „für welche unter den politischen Organen gerade kein Konsens zu finden war", lautet die nationalkonservative Position (Hilpold 2008, S. 26, Herv. i. O.), die hauptsächlich Kompetenzargumente vorbringt: „Die Gemeinschaft hat aber keine Kompetenz zur Schaffung einer Sozialunion" (Niemann 2004, S. 949). So laufe die Rechtsprechung des EuGH eindeutig dem Willen der Mitgliedsstaten entgegen, welche Schutzmechanismen gegen das „Risiko einer sozialleistungsinduzierten Freizügigkeit" etablieren wollten (Hailbronner 2004, S. 2187-2188). Die Konzeption des Allgemeinen der defensiv-hegemonialen Strategien lässt sich mit einer diskursiven Aussage bezeichnen, welche über die Jahre immer wieder zitiert wurde: die „nationale Wohlfahrts-Souveränität" (Fries u. Shaw 1998, S. 558*) - das fordistische Staatsprojekt.

Der EuGH folgt der offensiv-hegemonialen Strategie - am Anfang stark, im Verlauf der elf Jahre und der massiven Kritik der nationalen Projekte etwas zurückhaltender. Dies gelingt ihm vor allem durch die Konstruktion der Unionsbürgerschaft als Repräsentantin des Allgemeinen der offensiv-hegemonialen Strategie. Die RichterInnen entwickeln sie bereits im zweiten Rechtsfall (Grzelczyk), indem sie einen Vorschlag des Generalanwalts aus der ersten Rechtssache (Martínez Sala $)^{15}$ geringfügig modifiziert übernehmen:

„Der Unionsbürgerstatus ist nämlich dazu bestimmt, der grundlegende Status der Angehörigen der Mitgliedsstaaten zu sein, der es denjenigen unter ihnen, die sich in der gleichen Situation befinden, erlaubt, unabhängig von ihrer Staatsangehörigkeit [...] die gleiche rechtliche Behandlung zu genießen."16

Diese Definition wird in den folgenden Urteilen wie ein Mantra wiederholt. Sie inkludiert „grundlegend“ alle BürgerInnen der EU und verlangt ihre Gleichbehandlung unabhängig von ihrer Staatsbürgerschaft.

15 Schlussanträge des Generalanwalts Antonio Mario La Pergola vom 01. Juli 1997, Rdnr. 18.

16 Urteil, Rs. Grzelczyk (Fn. 15), Rdnr. 31. 


\section{3 Äquivalenzkette und antagonistische Zweiteilung}

Bei dem nächsten Kernstrategem handelt es sich um die Äquivalenzierung, also die Verknüpfung differenter, an dem jeweiligen Allgemeinen orientierter Positionen zu einer Äquivalenzkette. Der Gerichtshof verbindet über die Unionsbürgerschaft unterschiedliche weitere Rechtsnormen: vor allem das Diskriminierungsverbot aufgrund der Nationalität (Art. 12 EG), das spezielle Diskriminierungsverbot bei ArbeitnehmerInnen (Art. 39 EG), die Allgemeine Freizügigkeit (Art. $18 \mathrm{EG}$ ), aber auch die Nichterwerbstätigen-Richtlinien und die Freizügigkeitsrichtlinie 2004/38. Dabei ist das wesentliche juristische Argument immer, dass die Schaffung der Unionsbürgerschaft den „Anwendungsbereich“ der jeweiligen Norm erweitere. Sei zuvor eine bestimmte sozialpolitische Maßnahme (etwa für Arbeitsuchende oder für Studierende) noch nicht in die Kompetenz der EU gefallen, so ändere sich das durch die Einführung der Unionsbürgerschaft. Dafür korrigiert der Gerichtshof mehrfach sogar seine vorangegangene Rechtsprechung, in der er selbst die Nichtzuständigkeit festgestellt hatte. ${ }^{17}$ Ergebnis dieser Äquivalenzierungen ist jeweils ein eigenständiger vertraglicher Anspruch der nichterwerbstätigen UnionsbürgerInnen auf soziale Rechte, unabhängig davon, was die Richtlinien an Beschränkungen vorsahen. Dies erkannte der Gerichtshof auch für den Fall von Art. 18 EG an, obwohl die Freizügigkeit nur unter den „Bedingungen und Beschränkungen“ des Sekundärrechts gelten sollte. Diese änderten nichts an seiner Anwendbarkeit, sondern sagten lediglich etwas über die Ausübung aus.

Vor allem diese Argumentationsfigur rief immense Kritik der nationalen Projekte hervor: Kompetenzüberschreitung, methodisch fragwürdiges Vorgehen, „nachgerade skurrile“ Verdrehung des eindeutigen Wortlauts (Hailbronner 2004, S. 2187), politisches Sendungsbewusststein lauteten die Vorwürfe. „Auf welcher rechtsdogmatischen Grundlage der EuGH die Unionsbürgerschaft in ein Vehikel zur umfassenden Umwandlung der Europäischen Union in eine Solidargemeinschaft verwandelt, bleibt im Dunkeln “, war der Vorwurf des national-konservativen Projekts. Sie werde dadurch „zum kompetenzrechtlichen Zauberstab für die Verwirklichung der Vollintegration" (Hilpold 2008, S. 33). Dadurch dringe die „sozialpolitisch ambitionierte Rechtsprechung des EuGH [...] in den Bereich ,wohlfahrtstaatlicher Souveränität“" vor (Sander 2005, S. 1016) und die Mitgliedsstaaten verlören „weitgehend ihre Mitsprache- und Gestaltungsrechte“, während die Unionsbürgerschaft „zu einem absoluten Recht auf ,Sozialtourismus“" werde (Calliess 2007, S. 33-37).

In dieser manifesten Kritik kommt ein weiteres Kernstrategem zum Ausdruck: die antagonistische Zweiteilung des Raums. Denn die Herausbildung von Äquivalenzketten ist nicht nur über die positive Verknüpfung möglich, sondern muss durch die negative Abgrenzung zu all jenen diskursiven Elementen geschehen, zu denen sie in Kontrarität steht. Eine solche zweipolige Kontraritätsrelation „sucht jede hegemoniale Strategie zu instituieren“ (Nonhoff 2006, S. 216): in diesem Fall europäisch vs. national.

17 Etwa in der Rs. Collins, C-138/02, vom 23. März 2004, Slg. 2004, I-2703, Rdnr. 61 ff. 
Dies geschieht auch von der anderen Seite, den proeuropäischen Projekten, aus: Der Kritik wird vorgehalten, sie sei „heftig, aber unzutreffend“, missverstehe schlicht das Europarecht und unterstelle UnionsbürgerInnenschaft und Freizügigkeit lediglich deklaratorischen Charakter, wo sie doch eine konstitutive Wirkung besäßen, so die deutsche Generalanwältin beim EuGH, Juliane Kokott (2006, S. 219-220). Die Kritiker verkennten das „Verhältnis von bloß organisationsrechtlich bedeutsamer Kompetenzverteilung zur materiellen Grundrechtsgewährleistung “ (Rossi 2002, S. 352). Die „integrationsfreundliche Sozialrechtsprechung“ sei eindeutig „gegenüber staatlich gesteuerten Kampagnen“ zu verteidigen, sie müsse diesen gegenüber natürlich „als regelrechte Provokation erscheinen“ (Borchardt 2000, S. 10). Die Mitgliedsstaaten hätten die Unionsbürgerschaft selbst geschaffen, „und so sollten sie auch die Konsequenzen ihrer Entscheidung akzeptieren" (Iliopoulou u. Toner 2002, S. 619*).

Im Jahr 2006 gelang mit den Schlussanträgen von Kokott in der Rechtsache Tas/Tas-Hagen ${ }^{18}$ eine neue Äquivalenzierung, die auf eine ganz neue Weise hegemoniefähig war, sodass sie die Kritik kurzfristig verstummen ließ. Sie transplantierte nämlich in die Überlegungen zur Rechtsnatur des Art. 18 EG ein tragendes Argument der hegemonialen neoliberalen Grundfreiheitenjudikatur. Die klassischen Grundfreiheiten gälten „auch für diejenigen Sachgebiete, für die der Vertrag der Gemeinschaft keine eigenen Zuständigkeiten einräumt“. Denn andernfalls ließe sich „eine der Kernaufgaben der Gemeinschaft, die Verwirklichung eines Binnenmarktes [...], nicht sinnvoll verwirklichen. " 19 Und genauso widerspräche es dem Konzept der Unionsbürgerschaft als dem grundlegenden Status, wenn die Mitgliedsstaaten das in Artikel 18 Abs. 1 EG niedergelegte Freizügigkeitsrecht „nicht sachgebietsübergreifend beachten müssten “. ${ }^{20}$ Bis dato war umstritten, worum es sich bei Art. 18 überhaupt handelte: eine Grundfreiheit, ein Grundrecht oder etwas völlig Eigenes. Kokott, und darin folgte ihr der Gerichtshof ohne explizite begriffliche Benennung, brachte die Grundfreiheiten-These voran. Inzwischen wird relativ selbstverständlich von einer "Grundfreiheit ohne Markt" (Wollenschläger 2007, S. 125) beziehungsweise einer „neuen fünften Grundfreiheit“ (Fahey 2009, S. 949*) gesprochen. Kokott verknüpfte dadurch das tragende Diskurselement des hegemonialen neoliberalen Diskurses mit ganz anders gearteten, nicht der Binnenmarktlogik folgenden Diskurselementen der Unionsbürgerschaftsnormen. Dies ist eine wichtige Äquivalenzierungs-Strategie, was Laclau und Mouffe ([1985] 2000, S. 212) am genau gegenteiligen Fall aufgezeigt haben: nämlich wie sehr die erfolgreiche Verknüpfung einer Reihe von Kritiken sozialer Bewegungen am fordistischen Wohlfahrtsstaat mit dem neoliberalen Diskurs entscheidend für den Erfolg des Letzteren war.

18 C 192/05 Tas/Tas-Hagen, Slg. 2006, I-10451, vom 26.10.2006. Die NiederländerInnen Frau TasHagen und Herr Tas, die beide in Spanien lebten, waren als zivile Kriegsopfer anerkannt. Doch wurden ihnen durch die niederländischen Behörden die an diesen Status gebundenen Sozialleistungen aufgrund ihres spanischen Wohnsitzes verweigert.

19 Schlussanträge der Generalanwältin Juliane Kokott, vom 30. März 2006, Rdnr. 35.

20 Rdnr. 38. 


\subsection{Ausweichstrategem}

Der Gerichtshof vermied über eine bestimmte diskursive Argumentation, die ich Ausweichstrategem nennen möchte, eine eindeutige, in dem historischen Moment als zu aggressiv erscheinende Auslegung, indem er die im Zentrum stehende etablierte Rechtsfigur schlicht umging. Bereits in der ersten Rechtssache (Martínez Sala ${ }^{21}$ ) wich er der virulenten Diskussion darüber aus, ob Artikel 18 EG ein eigenständiges Freizügigkeitsrecht normiere (so die Kommission) oder nur die Wiederholung und Zusammenfassung bereits zuerkannter Rechte (so die Mitgliedsstaaten) sei, indem er gar nicht erst auf Art. 18 EG abstellte, sondern darauf, dass die Klägerin sich erlaubt in der BRD aufhalte, was von niemandem bestritten werde, sodass gar „nicht geprüft werden [muss], ob ihr Aufenthaltsrecht aus Art. 18 stammt". 22

Ausweichstrategien werden zumeist von den juridischen Intellektuellen durchschaut und wegen ihrer dogmatischen Inkonsistenz kritisiert - und zwar aus allen strategischen Richtungen. Zutreffend wurde hier vermutet, der Gerichtshof bereite damit „den Grund für eine spätere Auseinandersetzung mit Art. 18“ vor (Fries u. Shaw 1998, S. 546). Und tatsächlich, drei Urteile, drei Jahre später, in der Rs. Baumbast und R., ${ }^{23}$ schloss sich der EuGH der offensiv-hegemonialen Strategie an: Art. 18 Abs. 1 sei eindeutig und bedingungslos formuliert, daher komme ihm unmittelbare Wirkung zu. ${ }^{24}$ Das bedeutete, dass sich UnionsbürgerInnen unmittelbar auf Art. 18 Abs. 1 beziehen können, auf das Recht, sich im Hoheitsgebiet der Mitgliedsstaaten frei zu bewegen und aufzuhalten. ${ }^{25}$ Solche Beispiele für Ausweichstrategeme finden sich fast in jedem Urteil dieser Rechtsprechungslinie. Zumeist dreht es sich dabei um die Problematik der Vereinbarkeit der jeweils geltenden Richtlinien mit dem Primärrecht in der Rechtsprechung des EuGH. Der Gerichtshof ist bis zuletzt davor zurückgeschreckt, die Europarechtswidrigkeit der Richtlinien festzustellen, zugleich hat er sich jedoch auch nicht ihren restriktiven Vorgaben unterworfen, sondern die unterschiedlichsten „Bypässe“ (Brand 2005, S. 301) um die Richtlinien herum gebaut. Entweder hat er den Wortlaut der Richtlinie, insbesondere der Erwägungsgründe, in einer überraschenden Weise interpretiert, oder aber er hat, in der letzten Rechtssache aus 200926, die Freizügigkeitsrichtlinie „europarechtskonform“ ausgelegt, sodass dabei nur das nationale Umsetzungsgesetz als zu restriktive Interpretation der Richtlinie abgelehnt wurde.

21 (Fn. 2).

22 Rdnr. 60.

23 Rs. C-413/99 vom 17. September 2002, Slg. 2002, I-7091. In diesem Sachverhalt ging es nicht um soziale Rechte, denn die KlägerInnen waren wohlhabende UnternehmerInnen, sondern um die Frage, ob das Freizügigkeitsrecht in Art. 18 I EG einen eigenständigen Aufenthaltstitel gewährt. Diese Rechtssache brachte aber den Durchbruch für die Rechtsprechung zu den sozialen Rechten, da sie einen zweiten Begründungspfad etablierte.

24 Rn. 84 anschließend an die Schlussanträge des GA v. 05. Juli 1001, Rn. 103.

25 Urteil, Rn. 81.

26 Rs. Vatsouras/Koupatantze C-22/08 und C-23/08, Slg. 2009, I-0000, vom 04. Juni 2009, Rn. 44. Athanasios Vatsouras und Josif Koupatantze sind griechische Staatsbürger, die kurzfristig in der BRD beschäftigt waren, dann aber Leistungen für Arbeitssuchende nach SGB II beantragten. Diese wurden ihnen verweigert, da $\mathbb{} 7$ SGB II ausländische Arbeitssuchende von den Leistungen ausnahm. 
Ausweichstrategeme zeichnen sich dadurch aus, dass sie prekär sind, methodisch von allen diskursiven Positionen kritisiert werden und daher nur vorübergehende Überbrückungen darstellen.

\subsection{Zugeständnisstrategem}

Abschließend ist noch auf ein letztes ergänzendes Strategem zu verweisen, das eine besondere Form der Äquivalenzierung darstellt und das ich Zugeständnisstrategem nenne. Es trägt dem Umstand Rechnung, dass Hegemonie Zugeständnisse an die unterlegene Position voraussetzt, die so gravierend sind, dass sie es vermögen, diese asymmetrisch in das Projekt zu integrieren. Diskurstheoretisch werden so Elemente in den hegemonialen Diskurs hereingeholt, die bisher zur antagonistischen Gegen-Äquivalenzkette gehörten. Dieses Verfahren ist ein „zweischneidiges Schwert“, denn es erhöht zwar die Reichweite einer Hegemonie, lässt aber die antagonistische Grenze brüchig werden, sodass diese wieder mit beträchtlichem diskursivem Aufwand stabilisiert werden muss (Nonhoff 2006, S. 288).

Das Zugeständnisstrategem war ganz entscheidend im vorliegenden Diskurs, denn die massive Kritik an den anfänglichen Urteilen verdeutlichte, dass der EuGH nur von den proeuropäischen Hegemonieprojekten Zustimmung erhielt, während die nationalen Projekte massiv dagegen ,schossen'. Eine idealtypische Hegemonietechnik ist das Verhältnismäßigkeitsprinzip (vgl. Buckel u. Oberndorfer 2009, S. 292). Es steht am Schluss der Grundfreiheiten-Prüfung: Nachdem zunächst die Frage nach einem Eingriff in ein geschütztes Rechtsgut gestellt wurde, werden in der Verhältnismäßigkeitsprüfung, die sehr große Interpretationsspielräume bietet, das legitime Ziel dieses Eingriffes sowie seine Geeignetheit und Erforderlichkeit geprüft. Übersteht der meist nationale Rechtsakt diese Prüfung, heißt das, dass die individuellen KlägerInnen zwar ein vertraglich verankertes Recht besitzen, die nationale Regelung, die jenes soziale Recht verweigert, jedoch verhältnismäßig und damit europarechtskonform ist. Und genau über diese Prüfung integrierte der EuGH die meisten KritikerInnen diskursiv. In der Prüfung des legitimen Zieles erkannten zumeist die Generalanwälte ausdrücklich an, dass es legitim sei, Regelungen aufzustellen, die "Sozialtourismus“ verhindern sollen. ${ }^{27}$ Insbesondere könnten das Regelungen sein, die eine „tatsächliche Verbindung zum Arbeitsmarkt" des Aufnahmestaats ${ }^{28}$ beziehungsweise bei Studierenden ein Wohnsitzerfordernis verlangen. In fast allen Fällen ließen sich die KritikerInnen so ,einfangen'. Ihren größten diskursiven Triumph feierten sie $2008,{ }^{29}$ als der Gerichtshof ihnen, entgegen den Schlussanträgen des Generalanwaltes, weit entgegenkam und einen Fünfjahreszeitraum als Wohnsitzerfordernis für Studierende anerkannte, bevor diese Anspruch auf Sozialleistungen haben sollen. Dies wurde als endlich „akzeptabler Gesamtkompromiss“ anerkannt (Hilpold 2009, S. 43) und zudem als „Notbremse“ interpretiert (Papp 2009, S. 88).

27 Etwa in der Rs. Collins (Fn. 18), Schlussanträge des GA, Rdnr. 75.

28 Urteil, Rs. Collins, Rdnr. 67.

29 Rs. Förster, C-158/07, Slg. 2008, I-0000, vom 18. November 2008. 
Die Entwicklung eines Zugeständnisstrategems war ausschlaggebend für die Stabilisierung des Diskurses in den letzten Jahren. Dabei hat es die anfänglich sehr weitgehende Linie des EuGH in Richtung einer "Sozialunion“ allerdings auch verwässert und abgeschwächt. Doch kanalisierte die Verhältnismäßigkeitsprüfung die Kritik in die eigenen Diskursbahnen und behielt dabei das letzte Wort. Denn es ist der EuGH, der - zumeist gegen die Mitgliedsstaaten - die Geeignetheit und Erforderlichkeit einer Maßnahme prüft. Über dieses Prüfungsschema ließen sich die Kritiken vom ersten zentralen Prüfungspunkt, ob es überhaupt eine Anspruchsgrundlage im Europarecht gibt, hin zur Abwägung der Verhältnismäßigkeit umlenken und auf die bloße Ebene der Rechtfertigung überführen. Dort können in den Kritiken dann nur noch bereits vom Gericht akzeptierte Rechtfertigungsgründe vorgebracht werden, die aber auch jederzeit als unpassend zurückgewiesen werden können. Catherine Barnards empirische Studie hat gezeigt, dass in den beiden letzten Dekaden die Erfolgsrate von nationalen Rechtfertigungsversuchen gesunken ist (nach Shuibhne 2008, S. 784\%).

\section{Fazit}

Die Rechtsprechungslinie zu den sozialen Rechten Nichterwerbstätiger ist inzwischen etabliert, und die Präsenz der notwendigen Kernstrategeme zeigt an, dass sie in ein hegemoniales juridisches Projekt gemündet ist. Der EuGH konnte dabei sogar in den Entstehungsprozess der Freizügigkeits-Richtlinie intervenieren. Als Ergebnis „harmoniert die Richtlinie in vielen Aspekten augenfällig mit der Rechtsprechung" (Kokott 2006, S. 223). Obwohl die Mitgliedsstaaten in jeder Rechtssache eine zurückhaltende Auslegung forderten, unterließen sie es jedoch, im legislativen Prozess im Rat die Rechtsprechung wieder rückgängig zu machen oder aufzuhalten (vgl. Wissel 2010).

Dem Gerichtshof gelang es also, die Positionen der um Hegemonie kämpfenden Projekte zu organisieren und sie in ein eigenständiges juristisches Projekt einzubinden. Wie lässt sich dieses Projekt nun genauer bestimmen? Zunächst zur Rolle des EuGH: Generalanwalt Colomer erläutert, nicht etwa in einer philosophischen Abhandlung, sondern in den Schlussanträgen, sein Selbstverständnis der Rolle des Gerichtshofs: Es gebe drei Arten von RichterInnen:

„[...] die Handwerker, wahre Automaten, die mit bloßen Händen Urteile in Serie und in rauen Mengen produzieren, ohne in die Bereiche des Menschlichen oder der sozialen Ordnung hinabzusteigen, die Kunsthandwerker, die Hand und Hirn benutzen und sich dabei den traditionellen Auslegungsregeln unterwerfen, die sie unweigerlich dazu führen, den Willen des Gesetzgebers ohne Weiteres umzusetzen, und die Künstler, die mit Hilfe der Hände, des Kopfes und des Herzens den Bürgern weitere Horizonte eröffnen, ohne der Realität und den konkreten Sachverhalten den Rücken zu kehren.“30

Dass dieses Bild des „Künstler-Richters“, der sich von den in den politischen Prozessen zustande gekommenen Normen befreit, kaum Kritik erfahren hat, ob-

30 Schlussanträge des Generalanwalts D. Ruiz-Jarabo Colomer vom 20. März 2007, Rn. 1., in der Rs. Morgan/Bucher, Slg. 2007, I-9161, vom 23.10.2007. 
wohl die Gewaltenteilung zwischen Legislative und Judikative durchaus radikal in Frage gestellt wird, ist nur durch die Verselbstständigung der rechtlichen Bürokratie zu verstehen, die jedwede Kämpfe um Hegemonie überformt. Die Universalisierungsinstanz ist keine neutrale ,Relaisstation', sondern mischt jedem Ergebnis immer schon ihr eigenes Bestandserhaltsinteresse bei. Deswegen stützt der EuGH die proeuropäischen Hegemonieprojekte, denn die europäischen Institutionen sind in ständige Reskalierungs-Kämpfe verstrickt und daher bestrebt, die Stabilität und Kohärenz des europäischen Staatsapparate-Ensembles zu stärken (vgl. speziell zum EuGH Höreth 2008).

Dieses Bestandsinteresse ist aber immer vermittelt über die Fähigkeit, einen hegemonialen Konsens zu formulieren, um die eigene Legitimität aufrechtzuerhalten. Das heißt, nur indem sich der Gerichtshof auf die Diskurskoalition des proeuropäisch-sozialdemokratischen und linksliberal-alternativen Hegemonieprojekts stützte und in begrenztem Maße Zugeständnisse an die nationalen Projekte machte, gelang es ihm, seine Rechtsprechungskompetenz auszuweiten.

Entscheidend ist darüber hinaus die völlige Abwesenheit des neoliberalen Hegemonieprojekts. Zum einen zeigt sich darin bereits seine Schwächung - so ist nach wie vor sein diskursiver Ort die klassische Grundfreiheiten-Judikatur, obwohl sich längst eine fünfte, nicht binnenmarktbezogene Grundfreiheit herausgebildet hat. Allerdings ist dies nur die eine Seite der Medaille. Zugleich handelt es sich bei den hier geschaffenen transnationalen sozialen Rechten um keine vertikale, sondern nur um eine horizontale Umverteilung. Das neoliberale Projekt war insofern über seine Abwesenheit präsent: denn das so entwickelte juridische Projekt unterscheidet sich durch die diskursive Absenz des neoliberalen Hegemonieprojekts deutlich von demjenigen, welches der Gerichtshof seit Mitte der 1970erJahre im Bereich der Binnenmarktjudikatur organisierte.

Die diskursimmanente Erklärung dieses Bruchs lautet: In der „Post-Maastricht-Ratifizierungskrise“ sei die Notwendigkeit eines Identifikationsangebots für die europäischen BürgerInnen offensichtlich geworden, eines, das der EU eine „raison d'être gibt, die nicht nur rein ökonomisch sei. Dies wirft das Dilemma der Bürgerschaft auf" (Shaw 1998, S. 295*). Solange die EU exklusiv auf die ökonomische Integration fokussiert bleibe, sei sie „unfähig, die Unterstützung der Menschen Europas zu erlangen“, das demokratische wie auch das soziale Defizit seien hauptverantwortlich für das Desinteresse an der EU (Hatzopoulos 2005, S. 1601\%). „Es ist nicht verborgen geblieben, dass im Herzen des europäischen Integrationsprojekts eine kalte modernistische Leerstelle“ liege und der „Markt als Integrationstelos" zunehmend an Attraktivität verliere (Haltern 2005, S. 91). Diese Aussagen lassen sich als Selbstbeschreibungen des hegemonialen Diskurses über die sozialen Rechte rekonstruieren, die im folgenden Zitat aus der eingangs dargestellten Rechtssache ,Trojani‘ paradigmatisch zusammengefasst werden:

„Der frühere französische Staatspräsident Georges Pompidou hat die Europäische Gemeinschaft zu Beginn der 70er Jahre des vergangenen Jahrhunderts einmal etwas despektierlich als ,Gemeinschaft der Kaufleute und Krämer' bezeichnet. Vermutlich hätte er nicht im Traum daran gedacht, dass sein Landsmann Michel Trojani einmal zu europarechtlichem Ruhm gelangen würde. [Er] [...] dürfte in der Welt eines auf ökonomische Transaktionen fixierten Binnenmarktes nicht vorkommen. Doch diese 
Welt existiert so nicht mehr. [...] [Der EuGH] leistet damit einen weiteren wichtigen Beitrag zur Entkräftung der These, die das europäische Gemeinschaftsrecht als Bedrohung für die überkommene Sozialstaatlichkeit in den Mitgliedsstaaten ansieht. In einem ,Europa der Bürger' ist nämlich auch für einen armen Schlucker wie Michel Trojani Platz“ (Kingreen 2007, S. 74).

Das juridische Projekt lässt sich demnach in dieser Selbstbeschreibung nur als sozialpolitisches verwirklichen. Die massive Kritik, vor allem des proeuropäischsozialdemokratischen wie auch des national-sozialen Hegemonieprojekts, in der Folge des Binnenmarkts war nicht mehr zu ignorieren. Das heißt nun aber weder, dass die Effekte dieser Rechtsprechung tatsächlich eine Sozialunion konstituierten, noch dass die ,europäische BürgerInnenschaft ${ }^{\star}$ zunächst mehr als ein diskursives Konstrukt ist. In der Literatur wird sogar zum Teil vermutet, dass die „sozial-liberale" Rechtsprechung wegen der bloß individualrechtlichen Perspektive die gleichen Effekte habe wie die „wirtschaftsliberale“ (Scharpf 2008, S. 94). Hegemonietheoretisch steht einer europäischen Sozialunion vor allem entgegen, dass die beiden sozialen Hegemonieprojekte, die nur gemeinsam genügend Ressourcen mobilisieren könnten, in ein nationales und ein europäisches gespalten sind. Das national-soziale Projekt ist nicht Teil der proeuropäischen Diskurskoalition, sondern ist vielmehr eine solche mit dem national-konservativen eingegangen, wohingegen das proeuropäische gerade an den individualrechtlichen CitizenshipDiskurs des links-liberal-alternativen Hegemonieprojekts anknüpfte.

Es ist methodisch zwischen dem Diskurs und seinen Effekten, das heißt der Umsetzung des institutionell-normativen Programms in soziale Praxen zu unterscheiden. Diskursive Aussagen sind allerdings auch keine bloßen Repräsentationen, sondern sie wirken, und das ist ihre konstitutive, materielle Bedeutung, realitätsstiftend, weil sie Umrisse des Denk- und Sagbaren formieren, sie etablieren damit Möglichkeitsräume und Wiederholbarkeitsstrukturen. Indem sie zuallererst die Gegenstände, von denen gesprochen wird, konstituieren (Pieper 2006, S. 271), verfügen sie über eine „performative, wirklichkeitskonstituierende Macht“ (Keller 2004, S. 8). In diesem institutionalisierten rechtlichen Diskurs ist die neoliberale Hegemonie gebrochen und in ihren Ruinen hat sich eine neue hegemoniale Position entwickelt, die verlangt, dass die EU politisch-sozial und nicht bloß ökonomisch programmiert sein soll. Das darin zum Ausdruck kommende universelle Projekt einer Sozialunion wird durch die UnionsbürgerInnenschaft, das heißt einen genuin politischen Status repräsentiert. Jeder und jede einzelne BürgerIn der EU (und zugleich nur diese) können sich nunmehr zur Durchsetzung ihrer Rechte institutionell abgesichert darauf berufen. Damit werden ihre Rechte Teil der strategisch-selektiven Möglichkeitsstrukturen, an welche die Hegemonieprojekte anknüpfen können.

Étienne Balibar hat mit Bezug auf die Nation argumentiert, dass die Regulation der sozialen Kämpfe durch die Sozialpolitik seit dem Ende des 19. Jahrhunderts unentbehrlich für die Erhaltung der nationalen Form des Staates war - und zugleich diese Regulierung der sozialen Konflikte niemals ohne den Prozess der Einsetzung der Nation als Form privilegierter, sakralisierter Gemeinschaft möglich gewesen wäre. Das Ergebnis nennt er daher den „national-sozialen Staat“ (Bali- 
bar 2010, S. 25). Das juridische Projekt hat, kann man in Analogie zu Balibars Argument schlussfolgern, in der Krise der wettbewerbsstaatlichen Integrationsweise die ,europäisch-soziale Union` zum Inhalt. An diesem Punkt konvergiert es mit den politischen Strategien der Kommission (Wissel 2010).

„Die Europäische Union [...] ist auf der Suche nach einer politischen Imagination" (Haltern 2005, S. 111). Aber erst in dem Moment, da auch nationale Apparate in diese Strategie einstimmen oder von dieser eingehegt und überformt werden, kann sich ein Staatsprojekt entwickeln, also eines, welches in der Lage wäre, das europäische Staatsapparate-Ensemble zu einen. Ob dies gelingen wird oder nicht - gerade in der aktuellen Krisensituation -, hängt von der gesellschaftlichen Kräftekonstellation und den strategischen Praxen ab - die Suchprozesse in diese Richtung haben allerdings, das zeigt die Rechtsprechung zu den sozialen Rechten Nichterwerbstätiger, bereits begonnen.

\section{Literatur}

Balibar, Étienne. 2010. Kommunismus und (Staats-)Bürgerschaft. Überlegungen zur emanzipatorischen Politik. In Das Staatsverständnis von Nicos Poulantzas. Der Staat als gesellschaftliches Verhältnis, Hrsg. Alex Demirović, Stephan Adolphs und Serhat Karakayali, 19-34. Baden-Baden: Nomos.

Barnard, Catherine. 2005. Case C-209/03. Common Market Law Review 42:1465-1489. Bieling, Hans-Jürgen, und Jochen Steinhilber. 2000. Hegemoniale Projekte im Prozess der europäischen Integration. In Die Konfiguration Europas. Dimensionen einer kritischen Integrationstheorie, Hrsg. Hans-Jürgen Bieling und Jochen Steinhilber, 103-130. Münster: Wesfälisches Dampfboot.

Borchardt, Klaus-Dieter. 2000. Der sozialrechtliche Gehalt der Unionsbürgerschaft. Neue Juristische Wochenschrift 29/2000:2057-2061.

Brand, Michiel. 2005. Comments to Case-209/03 (Bidar). Columbia Journal of European Law 12:297-304.

Brand, Ulrich. 2011. State, Context and Correspondence. Contours of a Historical-Materialist Policy Analysis. Unveröffentlichtes Manuskript, Wien.

Buckel, Sonja. 2007. Subjektivierung und Kohäsion. Zur Rekonstruktion einer materialistischen Theorie des Rechts. Weilerswist: Velbrück Wissenschaft.

Buckel, Sonja, und Andreas Fischer-Lescano (Hrsg.). 2007. Hegemonie gepanzert mit Zwang. Zivilgesellschaft und Politik im Staatsverständnis Antonio Gramscis. BadenBaden: Nomos.

Buckel, Sonja, und Likas Oberndorfer. 2009. Die lange Inkubationszeit des Wettbewerbs der Rechtsordnungen. Eine Genealogie der Rechtsfälle Viking/Laval/Rüffert/Luxemburg aus der Perspektive einer materialistischen Europarechtstheorie. In Europäische Gesellschaftsverfassung. Zur Konstitutionalisierung sozialer Demokratie in Europa. Hrsg. Andreas Fischer-Lescano, Florian Rödl und Christoph Schmid, 277-296. BadenBaden: Nomos.

Buckel, Sonja, und Julia König. 2009. Körperwünsche im Recht - Zur Vermittlung von Rechtstheorie und Psychoanalyse. Kritische Justiz 42:337-352.

Calliess, Christian. 2007. Der Unionsbürger: Status, Dogmatik und Dynamik. Europarecht 42, Beiheft 1:7-42. 
Candela Soriano, Mercedes. 2005. Libre Circulación de Estudiantes y Ventajas Sociales. Comentario a la Sentencia del TJCE de 15 de marzo de 2005 en el asunto Bidar (C209/03). Revista General de Derecho Europeo 8:1-16.

Demirović, Alex. 2011. Materialist State Theory and the Transnationalization of the Capitalist State. Antipode 43:48-59.

Esser, Josef. 2008. Reflexionen über ein gestörtes Verhältnis. Materialistische Staatstheorie und deutsche Politikwissenschaft. In Der Staat der bürgerlichen Gesellschaft. Zum Staatsverständnis von Karl Marx, Staatsverständnisse, Bd. 18. Hrsg. Joachim Hirsch, John Kannankulam und Jens Wissel, 203-219. Baden-Baden: Nomos.

Fahey, Elaine. 2009. Interpretive Legitimacy and the Distinction Between 'Social Assistance' and 'Work Seekers' Allowance'. Comment on Vatsouras. European Law Review 34:933-949.

Fries, Sybilla, und Jo Shaw. 1998. Citizenship of the Union: First Steps in the European Court of Justice. European Public Law 4:533-559.

Gramsci, Antonio. 1991 ff. Gefängnishefte. Kritische Gesamtausgabe. Hrsg. Klaus Bochmann und Wolfgang Fritz Haug. Hamburg/Berlin: Argument Verlag.

Hailbronner, Kay. 2004. Die Unionsbürgerschaft und das Ende rationaler Jurisprudenz durch den EuGH? Neue Juristische Wochenschrift 57:2185-2189.

Haltern, Ulrich. 2005. Das Janusgesicht der Unionsbürgerschaft. Swiss Political Science Review 11:87-117.

Hatzopoulos, Vassilis. 2005. A (More) Social Europe: A Political Crossroad or a Legal One-Way? Dialogues Between Luxembourg and Lisbon. Common Market Law Review 42:1599-1635.

Hilpold, Peter. 2008. Unionsbürgerschaft und Bildungsrechte oder: Der EuGH-Richter als ,Künstler'. In Der EuGH und die Sonveränität der Mitgliedsstaaten. Eine kritische Analyse richterlicher Rechtsschöpfung auf ausgewählten Rechtsgebieten, Hrsg. Günter H. Roth und Peter Hilpold, 11-53. Wien: Boorberg.

Hilpold, Peter. 2009. Unterhaltsstipendien für Unionsbürger - Die Rechtssache ,Förster und die Grenzen mitgliedstaatlicher Solidarität. Europäische Zeitschrift für Wirtschaftsrecht 20:40-43.

Hirsch, Joachim. 2005. Materialistische Staatstheorie. Transformationsprozesse des kapitalistischen Staatensystems. Hamburg: VSA.

Hitzel-Cassagnes, Tanja. 2006. Der EuGH im Spannungsfeld von Konstitutionalisierung und Demokratisierung. In Politik und Recht, PVS-Sonderheft 36, Hrsg. Michael Becker und Ruth Zimmerling, 377-396. Wiesbaden: VS-Verlag für Sozialwissenschaften.

Höpner, Martin, und Armin Schäfer. 2008. Eine neue Phase der europäischen Integration: Legitimitätsdefizite europäischer Liberalisierungspolitik. In Die Politische Ökonomie der Europäischen Integration, Hrsg. Martin Höpner und Armin Schäfer, 129-156. Frankfurt a. M.: Campus Verlag.

Höreth, Marcus. 2008. Die Selbstautorisierung des Agenten. Der Europäische Gerichtshof im Vergleich zum U.S. Supreme Court. Baden-Baden: Nomos.

Iliopoulou, Anastasia, und Helen Toner. 2002. Case C-184/99. Common Market Law Review 39:609-620.

Jessop, Bob. 1990. State Theory. Putting the Capitalist State in its Place. Cambridge u. a.: Polity Press. 
Jessop, Bob. 2007. Althusser, Poulantzas, Buci-Glucksmann - Weiterentwicklungen von Gramscis Konzept des integralen Staats. In Hegemonie gepanzert mit Zwang. Zivilgesellschaft und Politik im Staatsverständnis Antonio Gramscis, Hrsg. Sonja Buckel und Andreas Fischer-Lescano, 43-65. Baden-Baden: Nomos.

Joerges, Christian. 2010. Europa nach dem Ordoliberalismus: Eine Philippika. Kritische Justiz 43:394-406.

Kannankulam, John, Fabian Georgi und Nikolai Huke. 2011 (im Erscheinen). Gesellschaftliche Kräfteverhältnisse und die europäische Unionsbürgerschaft: Eine historischmaterialistische Politikanalyse. FEI-Arbeitspapier Nr. 29. Marburg: Forschungsgruppe Europäische Integration, Phillips-Universität Marburg.

Keller, Reiner. 2004. Diskursforschung. Eine Einfübrung für SozialwissenschaftlerInnen. Wiesbaden: VS-Verlag für Sozialwissenschaften.

Kingreen, Thorsten. 2007. Die Universalisierung sozialer Rechte im europäischen Gemeinschaftsrecht. Europarecht 42, Beiheft 1: 43-74.

Kokott, Juliane. 2006. Die Freizügigkeit der Unionsbürger als neue Grundfreiheit. In Völkerrecht als Wertordnung. Festschrift für Christian Tomuschat, Hrsg. Pierre-Marie Dupuy, Bardo Fassbender, Malcom N. Shaw und Karl-Peter Sommermann, 207-226. Kehl/ Straßburg/Arlington: N. P. Engel Verlag.

Laclau, Ernesto, und Chantal Mouffe. [1985] 2000. Hegemonie und radikale Demokratie. Zur Dekonstruktion des Marxismus. Wien: Passagen Verlag.

Leibfried, Stephan, und Herbert Obinger. 2008. Nationale Sozialstaaten in der Europäischen Union: Zukünfte eines, sozialen Europas‘. In Die Politische Ökonomie der europäischen Integration, Hrsg. Martin Höpner und Armin Schäfer, 335-367. Frankfurt a. M.: Campus Verlag.

Lenaerts, Koen, und Tinne Heremans. 2006. Contours of a European Social Union in the Case-Law of the European Court of Justice. European Constitutional Law Review 2:101-115.

Lessenich, Stephan. 2009. Mobilität und Kontrolle. Zur Dialektik der Aktivgesellschaft. In Soziologie. Kapitalismus. Kritik. Eine Debatte, Hrsg. Klaus Dörre, Stephan Lessenich und Hartmut Rosa, 126-177. Frankfurt a. M.: Suhrkamp.

Letzner, Peggy. 2003. Sozialhilfe für Student aus anderem Mitgliedsstaat - EuGH, EUZW 2002. Juristische Schulung 52:118-122.

Ludwig, Gundula, Birgit Sauer und Stefanie Wöhl. 2009. Staat und Geschlecht: Grundlagen und aktuelle Herausforderungen. Eine Einleitung. In Staat und Geschlecht: Grundlagen und aktuelle Herausforderungen feministischer Staatstheorie, Hrsg. Gundula Ludwig, Birgit Sauer und Stefanie Wöhl, 11-27. Baden-Baden: Nomos.

Meulman, Jochen, und Henri de Waele. 2004. Funding the Life of Brian. Jobseekers, Welfare Shopping and the Frontiers of European Citizenship. Legal Issues of Economic Integration 31:275-288.

Neumann, Franz. 1937. Der Funktionswandel im Recht der bürgerlichen Gesellschaft. Zeitschrift für Sozialforschung 6:542-596.

Niemann, Ingo. 2004. Von der Unionsbürgerschaft zur Sozialunion? - Anmerkung zum Urteil des EuGH vom 23. März 2004, Rs. C-138/02 (Collins). Europarecht 6/2004:946-953.

Nonhoff, Martin. 2006. Politischer Diskurs und Hegemonie. Das Projekt „Soziale Marktwirtschaft". Bielefeld: Transcript. 
Papp v., Konstanze 2009. EuGH zieht Notbremse zum Schutz der sozialen Sicherungssysteme der Mitgliedstaaten bei Unterhaltsstipendien für Studenten. Neue Zeitschrift für Verwaltungsrecht. 2/2009:87-90.

Pieper, Marianne. 2006. Diskursanalysen - Kritische Analytik der Gegenwart und wissenspolitische Deutungsmuster. In Foucault: Diskursanalyse der Politik, Hrsg. Brigitte Kerchner und Silke Schneider, 269-286. Wiesbaden: VS-Verlag für Sozialwissenschaften.

Poulantzas, Nicos. [1978] 2002. Staatstheorie. Politischer Überbau, Ideologie, autoritärer Etatismus. Hamburg: VSA.

Rossi, Matthias. 2002. Anmerkung zum Grcelczyk-Urteil. Juristenzeitung 57:351-353.

Sander, Florian. 2005. Die Unionsbürgerschaft als Türöffner zu mitgliedsstaatlichen Sozialversicherungssystemen. Deutsches Verwaltungsblatt, 16/2005:1014-1022.

Scharpf, Fritz W. 2008. Individualrechte gegen nationale Solidarität. In Die Politische Ökonomie der europäischen Integration, Hrsg. Martin Höpner und Armin Schäfer, 89-99. Frankfurt a. M.: Campus Verlag.

Schönberger, Christoph. 2006. Die Unionsbürgerschaft als Sozialbürgerschaft. Zeitschrift für Ausländerrecht und Ausländerpolitik 26:226-231.

Shaw, Jo. 1998. The Interpretation of European Union Citizenship. The Modern Law Review 61:293-317.

Shuibhne, Niamh Nic. 2008. Case Law, Case C-76/05; C-318/05; C-11/06 \& C-12/06. Common Market Law Review 45:771-786.

Tömmel, Ingeborg. 2007. Governance und Policy-Making im Mehrebenensystem der EU. In Die Europäische Union - Governance und Policy-Making. Politische Vierteljahresschrift, Sonderheft Nr. 40, Hrsg. Ingeborg Tömmel, 13-36. Wiesbaden: VS-Verlag für Sozialwissenschaften.

Tomuschat, Christian. 2000. Comment on Case C-85/96, Maria Martínez Sala v. Freistaat Bayern, Judgement of 12 May 1998. Common Market Law Review 37:449-457.

van Apeldoorn, Bastiaan. 2009. The Contradictions of 'Embedded Neoliberalism' and Europe's Multi-Level Legitimacy Crisis: The European Project and its Limits. In Contradictions and Limits of Neoliberal European Governance. From Lisbon to Lisbon. Hrsg. Bastiaan van Appeldoorn, Jan Drahokoupil und Laura Horn, 21-43. Houndmils/ New York: Palgrave.

van der Mei, Anne P. 2005. Union Citizenship and the "De-Nationalisation" of the Territorial Welfare State. European Journal of Migration and Law 7:203-211.

von Bogdandy, Armin, und Stephan Bitter. 2005. Unionsbürgerschaft und Diskriminierungsverbot. Zur wechselseitigen Beschleunigung der Schwungräder unionaler Grundrechtsjudikatur. In Europa und seine Verfassung. Festschrift für Manfred Zuleeg zum 70. Geburtstag, Hrsg. Charlotte Gaitanides, Stefan Kadelbach und Gil Carlos Rodríguez Iglesias, 309-322. Baden-Baden: Nomos.

Weiler, Joseph H. H. 1996. The Selling of Europe: The Discourse of European Citizenship in the IGC 1996. Jean Monnet Working Paper 3/96. http://centers.law.nyu.edu/jeanmonnet/papers/96/9603.html. Zugegriffen: 20. Februar 2010.

Wiener, Antje. 2005. Bürgerschaft in neuen Grenzen: Zur sozialen Konstitution politischer Ordnung in Europa. In Grenzüberschreitungen. Differenz und Identiät im Europa der Gegenwart, Hrsg. Holger Huget, Chryssoula Kambas und Wolfgang Klein, 221-263. Wiesbaden: VS-Verlag für Sozialwissenschaften. 
Wind, Marlene. 2009. Post-National Citizenship in Europe: The EU as a "Welfare Rights Generator"? Columbia Journal of European Law 15:239-264.

Wissel, Jens. 2010. Staatsprojekt Europa. Der institutionelle Anfang. Abschlussbericht Fallstudie 1/Perspektive II, DFG-Projekt „Die Transnationalisierung des Staates im Prozess der Herausbildung einer gemeinsamen europäischen Migrationskontrollpolitik“. Manuskript, Frankfurt a. M., Oktober 2010.

Wollenschläger, Ferdinand. 2007. Grundfreiheit ohne Markt. Die Herausbildung der Unionsbürgerschaft im unionsrechtlichen Freizügigkeitsregime. Tübingen: Mohr Siebeck.

Ziltener, Patrick. 1999. Strukturwandel der europäischen Integration. Die Europäische Union und die Veränderung von Staatlichkeit. Münster: Westfälisches Dampfboot.

\section{Autorinnenangaben:}

Dr. Sonja Buckel

Institut für Sozialforschung, Senckenberganlage 26, 60325 Frankfurt am Main, s.buckel@soz.uni-frankfurt.de 